\title{
Oscillatory nonlinear conductance of an interacting quantum wire with an impurity
}

\author{
Fabrizio Dolcini ${ }^{1}$, Hermann Grabert ${ }^{1}$, Inès Safi ${ }^{2}$, and Björn Trauzettel ${ }^{1,2}$ \\ 1 Physikalisches Institut, Albert-Ludwigs-Universität, 79104 Freiburg, Germany \\ ${ }^{2}$ Laboratoire de Physique des Solides, Université Paris-Sud, 91405 Orsay, France
}

(Dated: August 8, 2003)

\begin{abstract}
The nonlinear conductance of a one-dimensional quantum wire adiabatically coupled to Fermi liquid electron reservoirs is determined in presence of an impurity. We show that electron-electron interaction in connection with the finite length of the wire leads to characteristic oscillations in the current as a function of the applied voltage.

PACS numbers: 71.10.Pm, 72.10.-d, 73.23.-b
\end{abstract}

It is well known that Fermi Liquid (FL) theory is not appropriate to describe transport properties of onedimensional (1D) electron systems. Correlation effects completely destroy Landau's quasiparticle picture, and the elementary excitations of a 1D quantumwire (QW) can be modelled in terms of bosonic plasmon and spin density modes. An equivalent representation employs fractionally charged quasiparticles [1, 2]. The charge ge depends on the parameter $g<1$ characterizing the electron-electron interaction strength, where $g=1$ corresponds to the non-interacting limit and $g \rightarrow 0$ means strong correlations. An impurity potential in a $1 \mathrm{D}$ QW is strongly renormalized by the interaction [3] and effectively acts as a back-scatterer of these fractionally charged quasiparticles [1].

These well known properties of 1D fermions are a natural consequence of the Luttinger Liquid (LL) model [4, 5], which generically describes the low energy physics of QWs. Despite these drastic departures from FL theory, the strong correlation effects are often disguised in transport measurements. For instance, the conductance of a clean QW is not affected at all by the electron-electron interaction within the wire [6, 7], and the shot noise of a wire with an impurity does not reflect the fractional charge of the backscattered quasiparticles 8, 9]. The reason lies in the coupling of the QW to the FL leads at its ends: experimentally only the chemical potential of electrons in the leads can be manipulated, while the chemical potential of the fractionally charged quasiparticles is not controllable. This represents the main difference with respect to the case of edge currents in fractional quantum Hall bars, where right and left movers are spatially separated (chiral LL), and the chemical potential of the Laughlin quasiparticles can be controlled by the upstream reservoir [10]. In contrast, in a semiconductor QW or a nanotube right and left movers interact and interfere in the same channel resulting in a nontrivial influence of the contacts even for the case of ideal adiabatic coupling.

Due to the important effects of the leads, a finite length QW in many respects acts as a complicated scatterer in a FL wire, and transport properties obey FL behavior. Thus, experimental observations of LL behavior in semiconductor QWs 11, 12] and nanotubes 13 are rather based on the temperature dependence of the conductance, or on modifications by the Coulomb interaction of resonant tunnelling through quantum dot structures
[14. Here we show that due to the interaction within the wire, the nonlinear conductance of a $\mathrm{QW}$ with a single impurity shows oscillatory behavior. In contrast to oscillations in double impurity systems 14 or in wires with tunneling contacts to the leads [15, 16], the oscillations predicted here are completely absent in a non-interacting wire. So we do not discuss modifications of an oscillatory effect already present in FL theory, but non-monotonic behavior of the conductance that is a signature of LL behavior.

To study a QW of finite length coupled to noninteracting reservoirs, we employ the inhomogeneous LL model [6, 7], where the interaction parameter has a spatial dependence. It varies from $g(|x|>L / 2)=1$ in the leads to $g(|x|<L / 2)=g<1$ in the QW. Although in real physical systems without backscattering at the contacts the variation of $g(x)$ from unity to $g$ must be smooth on a length scale $L_{s} \gg \lambda_{F}$, where $\lambda_{F}$ is the Fermi wavelength, it turns out that, as long as $L_{s} \ll L$, the simpler model of a $g(x)$ step-function already captures the important physical properties of a QW adiabatically connected to non-interacting electron reservoirs. Moreover, we have to keep in mind that only under the assumption $L_{s} \ll L$ the QW has a well-defined length $L$. Throughout this article we treat the spin-polarized situation at zero temperature, leaving finite temperature aspects for a future publication.

For $\hbar \equiv 1$, the full Lagrangian of our model is $\mathcal{L}=\mathcal{L}_{0}+$ $\mathcal{L}_{I}+\mathcal{L}_{U}$. In bosonization, see Ref. [5] for a recent review, the inhomogeneous LL Lagrangian of a clean system in terms of the phase field $\theta(x, t)$ reads

$$
\mathcal{L}_{0}=\frac{1}{2} \int d x\left[\frac{1}{v_{F}}\left(\partial_{t} \theta\right)^{2}-\frac{v_{F}}{g^{2}(x)}\left(\partial_{x} \theta\right)^{2}\right],
$$

where $v_{F}$ is the bare Fermi velocity of non-interacting right- and left-movers. The backscattering of an impurity at position $x_{0}$ yields the term

$$
\mathcal{L}_{I}=-\lambda \cos \left[\sqrt{4 \pi} \theta\left(x_{0}, t\right)+2 k_{F} x_{0}\right]
$$

with the 'bare' impurity strength $\lambda$, whereas the impurity forward scattering omitted here does not affect the current-voltage characteristics. A third term

$$
\mathcal{L}_{U}=-\frac{e}{\sqrt{\pi}} \int d x U(x) \partial_{x} \theta,
$$

describes the coupling to an electrostatic potential $U(x)$, with $e U(x<-L / 2)=\mu_{L}, e U(x>L / 2)=\mu_{R}$, 
and $U(x)=0$ for $|x|<L / 2$. Here, $\mu_{L}$ and $\mu_{R}$ are respectively the chemical potentials in the left and right reservoir, and we assume a symmetrically biased system with $\mu_{L}=e U / 2$ and $\mu_{R}=-e U / 2 . U(x)$ describes the shift of the band bottom due to electroneutrality in the FL leads [9]. In contrast, the QW itself does not remain electroneutral in presence of an applied voltage $U$ and the electrostatics within an interacting QW emerges naturally as a steady-state effect [9, 17].

In bosonization, the average current is given by $\langle I\rangle=$ $-(e / \sqrt{\pi}) \partial_{t}\langle\theta(x, t)\rangle$, and it is most convenient to decompose the field operator $\theta(x, t)$ as [17] $\theta(x, t)=\theta_{p}(x, t)+$ $\phi(x, t)$, where $\theta_{p}(x, t)$ is a particular solution of the equation of motion following from the action $\mathcal{S}[\theta]=$ $\mathcal{S}_{0}+\mathcal{S}_{I}+\mathcal{S}_{U}$ that corresponds to Eqs. (11) to (31). On the other hand, $\phi(x, t)$ can be regarded as the fluctuation operator which therefore has to fulfill

$$
\partial_{t}\langle\phi(x, t)\rangle=0 .
$$

Straightforward algebra shows that the particular solution may be written as

$$
\theta_{p}(x, t)=-\frac{e(U-V)}{2 \sqrt{\pi}} t+\left\{\begin{array}{rl}
\frac{e(U-V)}{2 \sqrt{\pi} v_{F}}|x| & , \quad|x|>\frac{L}{2} \\
-\frac{e g^{2} V}{2 \sqrt{\pi} v_{F}}|x| & , \quad|x|<\frac{L}{2}
\end{array} .\right.
$$

Consequently, the average current reads

$$
\langle I\rangle=\frac{e^{2}}{2 \pi}(U-V),
$$

where $V$ is a parameter corresponding to the fourterminal voltage drop at the impurity site. In order to determine $V$, one has to impose the condition (4) defining the fluctuation field $\phi$.
The effective action for $\phi$ is easily found to read

$$
\begin{aligned}
S[\phi] & =\frac{1}{2} \int d t \int d x\left[\frac{1}{v_{F}}\left(\partial_{t} \phi\right)^{2}-\frac{v_{F}}{g^{2}(x)}\left(\partial_{x} \phi\right)^{2}\right] \\
& -\frac{e V}{\sqrt{\pi}} \int d t \phi\left(x_{0}, t\right) \\
& -\lambda \int d t \cos \left[\sqrt{4 \pi} \phi\left(x_{0}, t\right)-e(U-V) t+2 k_{F} x_{0}\right]
\end{aligned}
$$

For weak backscattering the terms related to the impurity, namely the second and third line of Eq. (7), can be treated as perturbations on the free boson part (first line) employing standard techniques. The conditions when such a perturbative approach is reliable will be discussed below. It is convenient to characterize the conductance in terms of the backscattered current $I_{\mathrm{BS}}=I_{0}-\langle I\rangle=\left(e^{2} / 2 \pi\right) V$, where $I_{0}=\left(e^{2} / 2 \pi\right) U$. As can easily be shown, the lowest order contribution to $I_{\mathrm{BS}}$ is of order $\lambda^{2}$. Without loss of generality we assume $U>0$ and find, using the requirement (4), that the backscattered current takes the form

$$
I_{\mathrm{BS}}=\frac{e \lambda^{2} g L}{4 v_{F}} \int_{-\infty}^{\infty} d \tau e^{i u \tau} e^{4 \pi \mathcal{C}\left(\xi_{0}, \xi_{0}, \tau\right)}
$$

where $u=e U / \omega_{L}$ is the ratio of the voltage to the frequency $\omega_{L}=v_{F} / g L$ characterizing a ballistic traversal of the wire of length $L$. Here, the correlation function $\mathcal{C}(\xi, \eta, \tau)=\left\langle\phi(\xi, \tau) \phi(\eta, 0)-\phi^{2}(\eta, 0)\right\rangle_{0}$ in the absence of an impurity is expressed in terms of the dimensionless variables $\xi=x / L, \eta=y / L$ and $\tau=t v_{F} / g L$. At the dimensionless impurity site $\xi_{0}$ one can show that

$$
\mathcal{C}\left(\xi_{0}, \xi_{0}, \tau\right)=-\frac{g}{4 \pi}\left\{\sum_{m \in Z_{\text {even }}} \gamma^{|m|} \ln \left(\frac{(\alpha+i \tau)^{2}+m^{2}}{\alpha^{2}+m^{2}}\right)+\sum_{m \in Z_{\text {odd }}} \gamma^{|m|} \ln \left(\frac{(\alpha+i \tau)^{2}+\left(m-2 \xi_{0}\right)^{2}}{\alpha^{2}+\left(m-2 \xi_{0}\right)^{2}}\right)\right\}
$$

where $\gamma=(1-g) /(1+g)$, and $\alpha=\omega_{L} / \omega_{c}$. Here $\omega_{c}$ is the standard high-energy cutoff of the LL model with $\lambda$, $e U, \omega_{L} \ll \omega_{c}$.

Using Eqs. (8) and (9), the backscattered current can now be evaluated numerically for arbitrary values of the parameters $g$ (interaction strength), $u$ (voltage times length), and $\xi_{0}$ (impurity position). Fig. 11 depicts the result as a function of $u$ for two impurity positions (in the middle and at $1 / 4$ of $L$ ), and for an interaction value $g=0.25$ characteristic for single-walled metallic carbon nanotubes [18. Apparently, the backscattered current oscillates as a function of $u=e U / \omega_{L}$, and the oscillations die out as $u$ increases.
Analytical expressions for the current can be obtained in special situations. For a very long wire $(u \rightarrow \infty)$ one can perform an asymptotic expansion yielding

$$
I_{\mathrm{BS}}=I_{\mathrm{BS}}^{\mathrm{st}}(U)\left[1+f_{\mathrm{BS}}^{\mathrm{osc}}\left(u ; \xi_{0}\right)+\ldots\right]
$$

where $I_{\mathrm{BS}}^{\mathrm{st}}$ is the leading order term while $f_{\mathrm{BS}}^{\text {osc }}$ describes the dominant oscillating correction. The dots represent further subleading orders. Explicitly one finds

$$
I_{\mathrm{BS}}^{\mathrm{st}}(U)=\frac{\pi^{2} \mathcal{N}(\alpha ; g)}{\Gamma(2 g)} \frac{e^{2} U}{2 \pi} \frac{\left(\lambda / \omega_{c}\right)^{2}}{\left(e U / \omega_{c}\right)^{2(1-g)}},
$$

which is independent of the length $L$ of the wire and the impurity position, and exhibits a power-law behavior as a function of the applied voltage $U$ in accordance with 
the result for the homogeneous LL (see e.g. [3] ). $\mathcal{N}(\alpha ; g)$ is a constant of order unity. In contrast, the corrections explicitly depend on both $\xi_{0}$ and $L$ (through $u$ ), clearly showing that oscillations are a finite-size effect. For $0<$ $\left|\xi_{0}\right|<1 / 2$ the asymptotic expansion yields

$$
f_{\mathrm{BS}}^{\mathrm{osc}}\left(u ; \xi_{0}\right)=\frac{2 \Gamma(2 g)}{\Gamma(g \gamma)} \sum_{s= \pm} \mathcal{D}\left(g ; s\left|\xi_{0}\right|\right) \frac{\cos \left[\left(1-2 s\left|\xi_{0}\right|\right) u-\pi g(1+s \gamma / 2)\right]}{u^{2 g(1-\gamma / 2)}} \frac{\left(1+2 s\left|\xi_{0}\right|\right)^{2 g \gamma}}{\left(1-2 s\left|\xi_{0}\right|\right)^{g(2-\gamma)}\left(16\left|\xi_{0}\right|\right)^{g \gamma}}
$$

where $\mathcal{D}\left(g ; s \xi_{0}\right)$ is a numerical factor of order unity. A similar asymptotic expansion has been performed in Ref. 19], where the crossover from LL to FL behavior was studied for a tunnel barrier.

The two frequencies appearing in the cosine terms of Eq. (12) are related to the distances of the impurity from the two contacts (in units of the wire length $L$ ). The phase shifts $u_{s}=\pi g(1 \pm \gamma / 2)$ as well as the decay factors of the oscillations directly depend on the electronelectron interaction strength. Importantly, when the interaction in the wire is switched off $(g \rightarrow 1)$, i.e., when one deals with a homogeneous system, the oscillations vanish since $\Gamma(g \gamma) \rightarrow \infty$ in this limit. Clearly, $I_{\mathrm{BS}}$ also vanishes when there is no back-scatterer, i.e. $\lambda \rightarrow 0$.

One can understand the origin of these current oscillations in terms of interference effects of plasmon modes which are reflected both by the impurity and, in an Andreev-type process [6], by the wire-lead contacts. When varying either the length $L$ of the wire, or the voltage (which appears as a phase shift in the effective action (7)), one basically changes the interference conditions, which results in the oscillatory behavior of the current. The period of the oscillations is only independent of $g$ if the backscattered current is considered as a function of the dimensionless variable $u$; however, one has to keep in mind that $u\left(=e U g L / v_{F}\right)$ has an intrinsic $v_{F} / g$-dependence.

We also point out that the expression (12) is singular for $\xi_{0} \rightarrow 0$; this is just due to a mathematical complication when the distances of the impurity from the two contacts become equal. Then pairs of poles in the correlator merge. The full expression (8) for the current is however perfectly regular, and indeed one can still calculate the asymptotic expansion for this particular impurity position, obtaining

$f_{\mathrm{BS}}^{\mathrm{osc}}=\frac{\Gamma(2 g) 2^{1-2 g \gamma}}{\Gamma(2 g \gamma)} \frac{\cos [u-\pi g(1+\gamma)]}{u^{2 g(1-\gamma)}} \prod_{n=2}^{\infty}\left(\frac{n^{2}}{n^{2}-1}\right)^{2 g \gamma^{n}}$

It should be emphasized that in the asymptotic expansion (10) the further subleading orders (represented by dots) can be shown to become increasingly important the stronger the interaction is, i.e., the lower the value of $g$. In practice, retaining only $I_{\mathrm{BS}}^{\mathrm{st}}$ and $f_{\mathrm{BS}}^{\mathrm{osc}}$ is a very good approximation for $g \gtrsim 0.5$ and $u \gtrsim 10$. Nevertheless, also for $g<0.5$ and/or lower values of $u$ (not too close to $u=1$ ), Eq. (10) gives a qualitatively correct picture of the properties of the backscattered current as we have checked numerically.

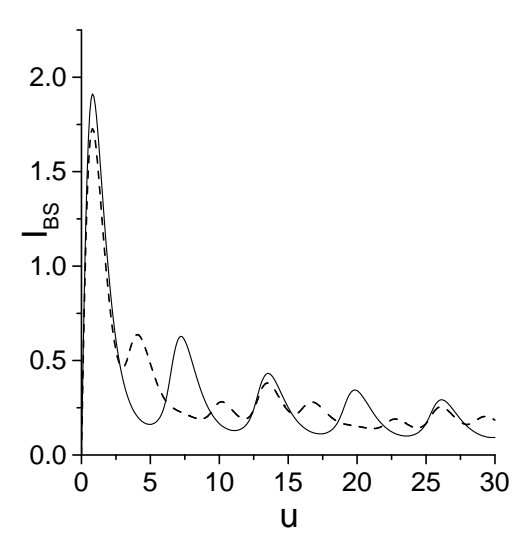

FIG. 1: The backscattered current $I_{\mathrm{BS}}$ is depicted, in units of $e\left(\lambda \omega_{L}^{g} / \omega_{c}^{g}\right)^{2} / \omega_{L} \mathcal{N}(\alpha ; g)$, as a function of $u=e U / \omega_{L}$. The interaction strength is $g=0.25$. The solid line corresponds to $\xi_{0}=0$ and the dashed line to $\xi_{0}=0.25$. Decaying oscillations with a constant period are clearly visible for the case $\xi_{0}=0$.

We now discuss how the behavior of the backscattered current changes with the interaction strength $g$. In Fig. 2 the full numerical result of $I_{\mathrm{BS}}$, Eq. (8), is plotted in units of $I_{\mathrm{BS}}^{\mathrm{st}}, \mathrm{Eq}$. (11), for three different values of $g$. For simplicity we have considered the case of an impurity located in the middle. The figure illustrates that the stronger the electron-electron interaction, the more pronounced the oscillations are. This is due to the fact that the Andreev-type reflections at the contacts are interactiondependent with a reflection coefficient $\gamma=(1-g) /(1+g)$ 6]. Thus, interference effects are most pronounced in the limit $g \rightarrow 0$. Furthermore, we see that the period of the oscillations is the same for any value of $g$, namely $\Delta u=2 \pi$ for $\xi_{0}=0$, but different values of $g$ yield different phase shifts.

In the opposite limit of a short wire $(u \rightarrow 0)$, the integral (8) can also be evaluated analytically, and we find for the backscattered current

$$
I_{\mathrm{BS}}=\mathcal{R}(\lambda, g) \frac{e^{2}}{2 \pi} U
$$

with the cut-off and interaction dependent 'reflection coefficient'

$$
\mathcal{R}(\lambda, g)=\pi^{2} \mathcal{N}_{2}(\alpha ; g) \frac{\left(\lambda / \omega_{c}\right)^{2}}{\left(\omega_{L} / \omega_{c}\right)^{2(1-g)}}
$$

where the constant $\mathcal{N}_{2}(\alpha ; g)$ is of order unity. The power 


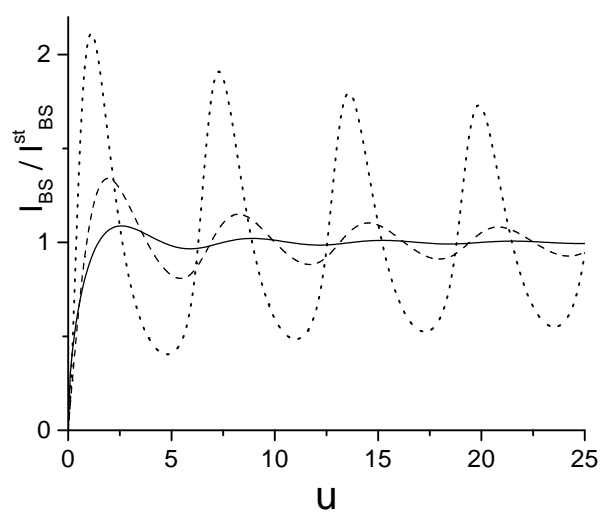

FIG. 2: The backscattered current $I_{\mathrm{BS}}$ is plotted, in units of $I_{\mathrm{BS}}^{\mathrm{st}}$, as a function of $u=e U / \omega_{L}$ for three different values of the interaction strength: $g=0.25$ (dotted line), $g=0.50$ (dashed line), and $g=0.75$ (solid line). Electron-electron interaction affects the decay as well as the phase shift of the oscillations.

law behavior of $\mathcal{R}(\lambda, g)$ as a function of the system size $L$ agrees with the calculation of the linear conductance from the Kubo formula [20]. Furthermore, Eq. (13) bears a resemblance to the usual Landauer formula. In the limit $g \rightarrow 1$ the reflection coefficient approaches the expected value $\mathcal{R}(\lambda, 1)=\pi^{2}\left(\lambda / \omega_{c}\right)^{2}$.

Finally we discuss the applicability of the perturbative approach. To be consistent, the backscattered current $I_{\mathrm{BS}}$ has to be small compared to the current $I_{0}$ in the absence of an impurity, i.e.,

$$
\left|I_{\mathrm{BS}}\right| \ll e^{2} U / 2 \pi .
$$

The analytical expressions obtained in the limits of both large $u$, Eqs. (10)-(11), and small $u$, Eqs. (13)-(14), suggest that the consistency condition can be expressed in a simple and cut-off independent way, if we introduce an effective impurity strength $\lambda^{*}=\omega_{c}\left(\lambda / \omega_{c}\right)^{1 /(1-g)}$. Indeed, Eq. (15) then amounts to $\lambda^{*} \ll e U$ for large $u$, and $\lambda^{*} \ll \omega_{L}$ for small $u$, respectively. This is in accordance with general reasoning. It is well known that the backscattering term (21) is a relevant perturbation to the LL fixed point, and therefore the RG-flow would drive the system to two disconnected QWs, unless some energy-scale cuts off the flow of $\lambda$. In our case the applied voltage $U$ or the wire ballistic frequency $\omega_{L}$ can play such a role. Therefore, as long as $\lambda^{*}$ is much smaller than either $e U$ or $\omega_{L}$, the QW remains in the neighborhood of the LL fixed point and our perturbative treatment is justified. In the limit $g \rightarrow 1$, the effective impurity strength $\lambda^{*}$ vanishes, and perturbation theory is always applicable if $\lambda \ll \omega_{c}$.

In summary, we have investigated the backscattered current of an interacting QW of finite size $L$ that is coupled to non-interacting electron reservoirs. We have found that the interplay between electron-electron interaction and finite size gives rise to distinct features in the current-voltage characteristics. In particular, we have found an oscillation of the backscattered current as a function of $u=e U / \omega_{L}$. For carbon nanotubes one can estimate a typical ballistic frequency of the order of a few meV. From Figs. 11and 2 one can see that the oscillations are visible for $e U \sim 10 \ldots 50 \mathrm{meV}$, a reasonable value, which is consistent with the cut-off of about $1 \mathrm{eV}$. Hence, the predicted effects should be observable.

We thank H. Bouchiat, R. Egger, D. C. Glattli, and W. Häusler for helpful discussions. Financial support by the CNRS, the DFG and the EU and are gratefully acknowledged.
[1] M. P. A. Fisher and L. I. Glazman in Mesoscopic Electron Transport, Vol. 345 of NATO ASI, edited by L. Kouwenhoven, G. Schön, and L. Sohn (Kluwer, Dordrecht, 1997), p.331.

[2] K.-V. Pham, M. Gabay, and P. Lederer, Phys. Rev. B 61, 16397 (2000).

[3] C. L. Kane and M. P. A. Fisher, Phys. Rev. B 46, 15233 (1992).

[4] F. D. M. Haldane, J. Phys. C: Solid State Phys. 14, 2585 (1981).

[5] A. O. Gogolin, A. A. Nersesyan, and A. M. Tsvelik, Bosonization and Strongly Correlated Systems (Cambridge University Press, Cambridge, 1998).

[6] I. Safi and H. J. Schulz, Phys. Rev. B 52, R17040 (1995).

[7] D. L. Maslov and M. Stone, Phys. Rev. B 52, R5539 (1995); V. V. Ponomarenko, ibid. 52, R8666 (1995).

[8] V. V. Ponomarenko and N. Nagaosa, Phys. Rev. B 60, 16865 (1999).

[9] B. Trauzettel, R. Egger, and H. Grabert, Phys. Rev. Lett. 88, 116401 (2002).

[10] F. P. Milliken, C. P. Umbach, and R. A. Webb, Solid State Comm. 97, 309 (1996).
[11] S. Tarucha, T. Honda, and T. Saku, Solid State Comm. 94, 413 (1995).

[12] A. Yacoby, H. L. Stormer, N. S. Wingreen, L. N. Pfeiffer, K. W. Baldwin, and K. W. West, Phys. Rev. Lett. 77, 4612 (1996).

[13] C. Dekker, Physics Today 52(5), 22 (1999).

[14] H. W. C. Postma, T. Teepen, Z. Yao, M. Grifoni, and C. Dekker, Science 293, 76 (2001).

[15] W. Liang, M. Bockrath, D. Bozovic, J. H. Hafner, M. Tinkham, and H. Park, Nature 411, 665 (2001).

[16] C. S. Peca, L. Balents, and K. J. Wiese, cond-mat/0304496 (2003).

[17] R. Egger and H. Grabert, Phys. Rev. Lett. 77, 538 (1996); 80, 2255(E) (1998); ibid. 79, 3463 (1997).

[18] R. Egger and A. O. Gogolin, Phys. Rev. Lett. 79, 5082 (1997); C. Kane, L. Balents, and M. P. A. Fisher, ibid. 79, 5086 (1997).

[19] V. V. Ponomarenko and N. Nagaosa, Phys. Rev. B 56, R12756 (1997).

[20] I. Safi and H. J. Schulz, in Quantum Transport in Semiconductor Submicron Structures, Vol. 324 of NATO ASI, edited by B. Kramer (Kluwer, Dordrecht, 1995), p. 159; 
Phys. Rev. B 59, 3040 (1999). 